\title{
Estimating friction factors while drilling
}

\author{
Ulf Jakob F. Aarsnes ${ }^{\mathrm{a}, *}$, Jean Auriol ${ }^{\mathrm{b}}$, Florent Di Meglio ${ }^{\mathrm{c}}$, Roman J. Shor ${ }^{\mathrm{b}}$ \\ ${ }^{a}$ NORCE Norwegian Research Centre AS, Oslo, Norway \\ ${ }^{b}$ University of Calgary, Department of Chemical and Petroleum Engineering, Calgary, Canada \\ ${ }^{c}$ Centre Automatique et Systemes, Mines ParisTech, Paris, France
}

\begin{abstract}
We design and test an algorithm for estimating the bit angular velocity and side forces on a drill string using only topside measurements fed into an adaptive observer. To derive the algorithm gains the drill string is modeled as a distributed wave equation coupled with Ordinary Differential Equations (ODEs) at the boundaries which represent the lumped dynamics of the top-drive and BHA, respectively. Here, the algorithm uses only measurements from the top-drive, while the non-linear side-forces are lumped at the BHA. The obtained observer gains are then implemented on a previously validated torsional drill string model with a distributed BHA model and distributed Coulomb side forces implemented as an inclusion. This design approach combines a high fidelity model with recent theoretical developments on estimation of PDEs to find appropriate feedback gains to ensure a fast and robust tuning. The feasibility of the approach is illustrated by showing convergence in estimated friction factors to the true values when testing on simulated data. When testing on full scale field data, friction factors converge to the same value for different initial values which indicates both good estimates and a robustness of the approach. The estimates produced by the algorithm can be displayed to a driller in real time in an advisory system, and the result can be built on to help optimize the drilling operation, detect faults and unwanted incidents, aid on-site decision making, and improve control of directional drilling.
\end{abstract}

\section{Introduction}

Exploration and production of oil and gas reserves in the deep subsurface necessitates the precise placement of a narrow wellbore, between 10 and $50 \mathrm{~cm}$ diameter, of lengths up to $10,000 \mathrm{~m}$ through a varied subsurface geological environment. Modern wellbores are rarely straight and typically must follow preplanned wellplans, ranging from simplier horizontal or deviated wells to complex three dimensional paths. In these complex wellbores, torque and drag due to friction between the drillstring and borehole wall become significant and increase the complexity of steering operations. Steering is typically achieved through the use of rotary steerable systems - which use actuated downhole tools to physically point or push the bit in a predefined direction - or bent motor assemblies - which use a permanent bend in a downhole mud motor to impart a deviation in hole angle if the motor bend is held in a fixed orientation. The latter system is often used for land wells due to its low cost, but presents challenges for the directional drillers due the complexity of the drillstring dynamic behavior and uncertainty in borehole torque and drag modelling.

Drillstring dynamic behavior often manifests itself as drill string vibrations, and has been extensively studied.

\footnotetext{
* Corresponding Author

Email address: ulaa@norceresearch.com (Ulf Jakob F. Aarsnes)
}

In particular, the torsional oscillations known as stick slip are considered to be one of the most prevalent vibrations. These stick-slip oscillations are characterized by a series of stopping - "sticking" - and releasing - "slipping" events of the bit. These models, if properly formulated, lend themselves to estimation problems and can be used to estimate friction parameters, and in turn, directional abilities of the drilling system.

A distributed model was used to characterize stick slip oscillation as caused by Coulomb friction induced side forces in [1]. Such stick slip can also occur off-bottom, and does not require a velocity weakening in the bit-rock interaction. Off-bottom stick slip is a well known phenomenon from the field, and when mentioned in literature is hypothesized to be caused by a negative difference between static and kinetic along-string Coulomb-type friction [8, 15, 21]. This cause is distinct [4] from stick-slip caused by the regenerative effect in the bit-rock interaction $[6,11,18]$.

The distributed effects of the drill string becomes an increasingly prominent feature of torsional dynamics in long wells, and perhaps especially wellbores with highinclination laterals. The nonlinear nature of the Coulomb friction can excite a wide range of frequencies where higher order modes become essential for representing the dynamics of the system, in particular for long wells. Hence, lumped approximations of the drill string easily fall short, and it is desirable to employ distributed model representations of the torsional dynamics, along the lines of $[2,5$, $14,1]$. 
The approach taken in the present work is to use a distributed model of the drill string with the lumped representation of the BHA used in [1] where the Coulomb friction is given as a source term implemented as an inclusion, with the states propagated according to the Filippov solution [13]. The distributed nature of the model enables it to capture a large part of the possible dynamics for a significantly extended range of well lengths and surveys compared to the more standard lumped approximations. At the same time, the relative mathematical simplicity of the formulation, a 1-D wave equation with a source term with ODEs as boundary conditions, enables us to employ new results from control theory [12] to effectively derive a soft sensor as an Luenberger Observer. Using only topside measurements, this soft sensor can in turn estimate kinetic and static friction factors as well as the distributed states of the system including drill string angular velocity and angular strain. This have several applications

1. Effectively estimating static and kinetic friction factors while drilling can help optimize the drilling operation, detect faults and unwanted incidents and aid on-site decision making.

2. An adaptive on-line observer opens up several promising new venues for avoiding torsional oscillations.

3. Improved estimates of drill string orientation have important applications for directional drilling [19, 9].

This paper begins with a derivation of the torsional drillstring model in Section 2, including validation with two sets of field data. The semi-lumped approximation needed for observer design is then given in Section 3, and the observer design itself in Section 4. Finally, in Section 5 , convergence behavior of the observer is demonstrated on both synthetic and field data.

\section{Model}

In this section we present the model under consideration. The validity of the proposed model is illustrated by considering the open-loop fit of the model to full scale field data at the end of the Section.

\subsection{Torsional dynamics of drill string}

The distributed model we use in this paper is similar to the one proposed in $[6,2,14]$, the main difference being that we only consider here the torsional dynamics. That is, for the angular motion, we denote the angular velocity and torque as $\omega(t, x), \tau(t, x)$ which are functions of $(t, x)$ evolving in $\{(t, x) \mid \quad 0<t<T, \quad x \in[0, L]\}$ (where $L$ is the total length of the drill string and $T$ a positive time), see Fig. 1. The torque is found from shear strain, given as twist per unit length. Letting $\phi$ denote the angular displacement (see Fig. 2) in the string s.t. $\frac{\partial \phi(t, x)}{\partial t}=\omega(t, x)$, we have $\tau(t, x)=J G(\phi(t, x)-\phi(t, x+d x)) / d x$, where $J$ is
Table 1: Nomenclature

\section{Parameters}

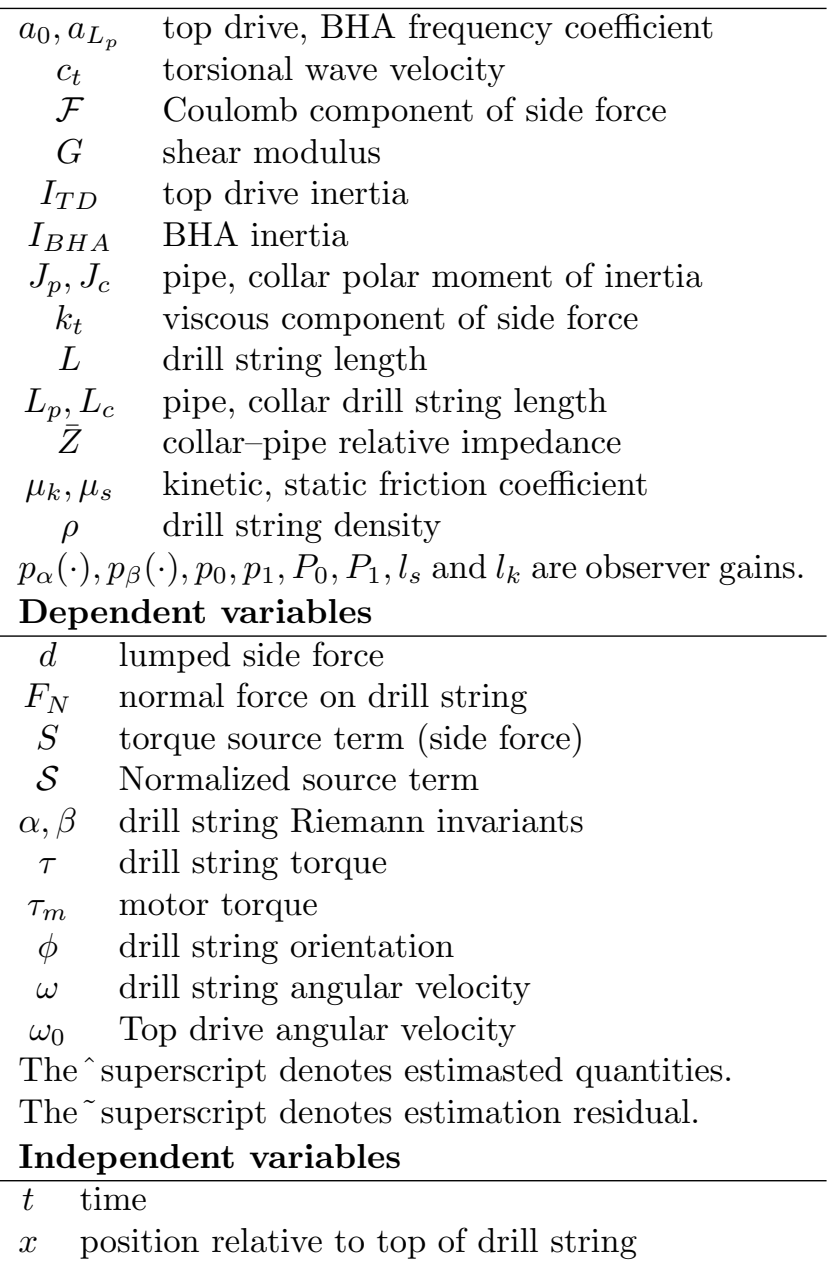




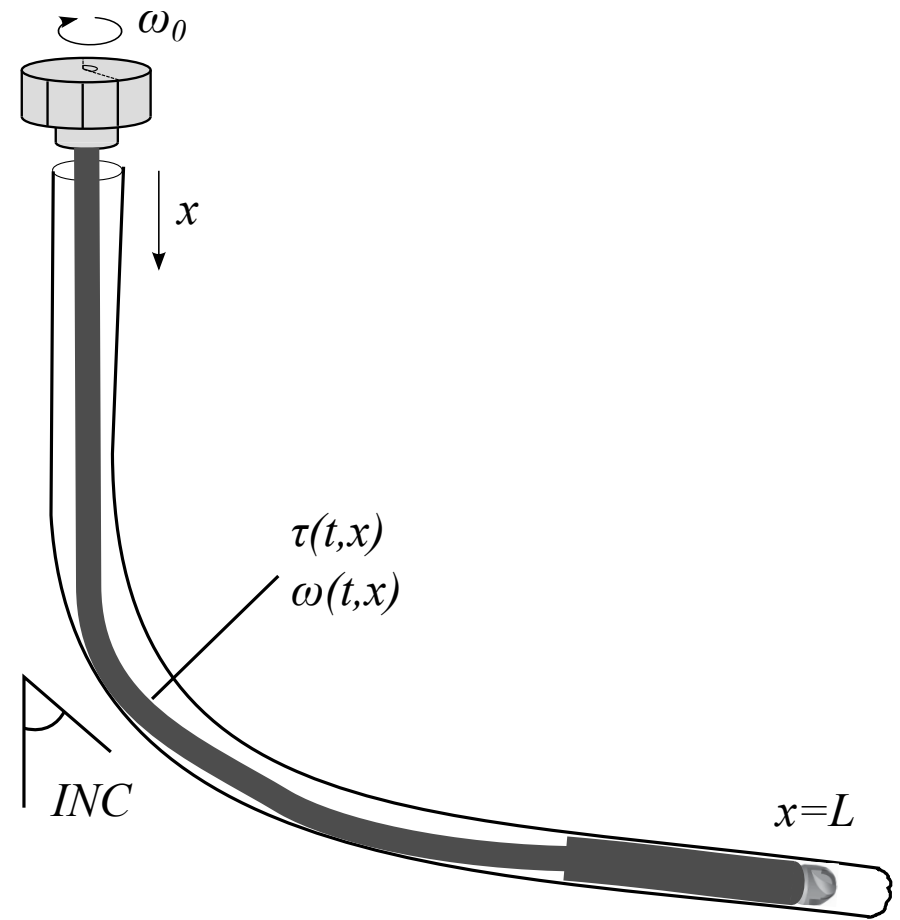

Figure 1: Schematic indicating the distributed drill string lying in deviate borehole.

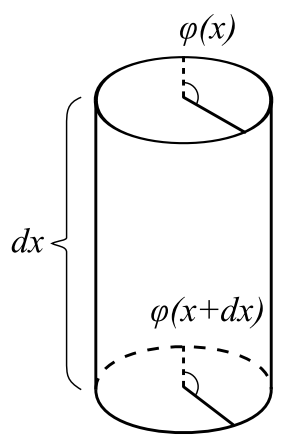

Figure 2: Infinitesmal drill string element.

the polar moment of inertia and $G$ is the shear modulus. Hence the equations for the angular motion are given by

$$
\begin{aligned}
& \frac{\partial \tau(t, x)}{\partial t}+J G \frac{\partial \omega(t, x)}{\partial x}=0, \\
& J \rho \frac{\partial \omega(t, x)}{\partial t}+\frac{\partial \tau(t, x)}{\partial x}=S(t, x),
\end{aligned}
$$

where the source term is due to frictional contact with the borehole and is modeled as

$$
S(t, x)=-k_{t} \rho J \omega(t, x)-\mathcal{F}(\omega, \tau, x)
$$

where $k_{t}$ is a constant damping term representing the viscous shear stresses between the pipe and drilling mud, and $\mathcal{F}(\omega, \tau, x)$ is a differential inclusion, that still has to be described, representing the Coulomb friction between the drill string and the borehole.

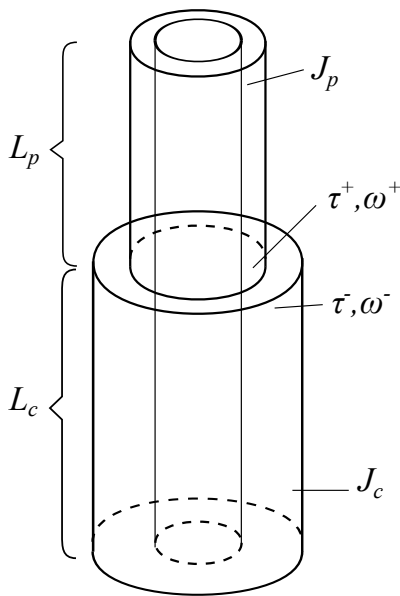

Figure 3: Collar-Pipe transition.

\subsection{Discontinuities of a multiple sectioned drill string}

The lowermost section of the drill string is typically made up of drill collars which may have a great impact on the drill string dynamic due to their added inertia. In particular, the transition from the pipes to collars in the drill string will cause reflections in the travelling waves due to the change in characteristic line impedance [2].

We split the drill string into a pipe section with polar moment of inertia and lengths $J_{p}, L_{p}$ and a collar section with the same parameters given as $J_{c}, L_{c}$. We use $\tau^{+}, \omega^{+}$ to denote the strain and velocity at the top of the drill collar and $\tau^{-}, \omega^{-}$at the bottom of the pipe, see Fig. 3 . The boundary conditions at the transition are given by the following continuity constraints

$$
\omega^{+}=\omega^{-}, \quad \tau^{+}=\tau^{-} .
$$

\subsection{Coulomb friction as an inclusion}

The Coulomb friction is modeled as an inclusion

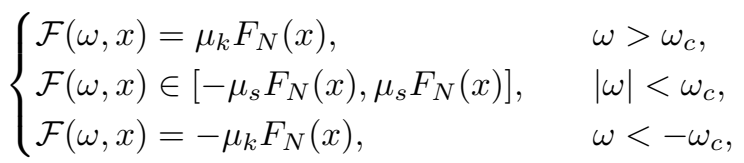

where $F_{N}$ is the normal force acting between the drill string and the borehole wall, $\omega_{c}$ is the threshold on the angular velocity where the Coulomb friction switches from static to kinetic, $\mu_{s}, \mu_{k}$ are the static and kinetic friction factors, respectively. Here, we distinguish between the kinetic and static friction factors: Static friction is friction between two or more solid objects that are not moving relative to each other. Kinetic friction, also known as dynamic friction or sliding friction, occurs when two objects are moving relative to each other and rub together. The function $\mathcal{F}(\omega, \tau, x) \in\left[-\mu_{s} F_{N}(x), \mu_{s} F_{N}(x)\right]$ denotes the inclusion where

$$
\begin{aligned}
\mathcal{F}(\omega, \tau, x)= & -\frac{\partial \tau(t, x)}{\partial x} \\
& -k_{t} \rho J \omega(t, x) \in\left[-\mu_{s} F_{N}(x), \mu_{s} F_{N}(x)\right],
\end{aligned}
$$


and take the boundary values $\pm \mu_{s} F_{N}(x)$ if this relation does not hold. The normal force profile of the drill string should be obtained from an appropriate torque and drag model, see e.g. [17, 20, 1].

\subsection{Boundary condition}

At the topside boundary, the top drive is actuated by a motor delivering a torque $\tau_{m}$ which we assume to be the control input. The topdrive has the inertia $I_{T D}$ and hence satisfies the dynamics

$$
\frac{\partial \omega_{0}}{\partial t}=\frac{1}{I_{T D}}\left(\tau_{m}-\tau(t, x=0)\right)
$$

and finally, the angular velocity at the top of the drill string is equal the top drive velocity $\omega(t, x=0)=\omega_{0}$.

\subsection{Derivation of Riemann invariants}

The Riemann invariants of a Hyperbolic PDE are the states corresponding to a transformation of the system which has a diagonalized transport matrix, i.e. the system can be written as a series of transport equations only coupled through the source terms $[16]$. On the set $\{(t, x) \mid \quad 0<$ $t<T, \quad x \in[0, L]\}$, we define the Riemann invariants as

$$
\alpha=\omega+\frac{c_{t}}{J G} \tau, \quad \beta=\omega-\frac{c_{t}}{J G} \tau,
$$

where $c_{t}=\sqrt{\frac{G}{\rho}}$ is the velocity of the torsional wave. This transformation enables us to rewrite (1)-(2) in variables $\alpha, \beta$ as the diagonalized PDE system

$$
\begin{aligned}
& \frac{\partial \alpha}{\partial t}(t, x)+c_{t} \frac{\partial \alpha}{\partial x}(t, x)=\mathcal{S}(t, x), \\
& \frac{\partial \beta}{\partial t}(t, x)-c_{t} \frac{\partial \beta}{\partial x}(t, x)=\mathcal{S}(t, x) .
\end{aligned}
$$

where the source term $\mathcal{S}$ is defined by

$$
\mathcal{S}(t, x)=\frac{S(t, x)}{J \rho}=k_{t}(\alpha(t, x)+\beta(t, x))+\frac{1}{J \rho} \mathcal{F}(t, x),
$$

where $\mathcal{F}$ is the inclusion given in (5) (expressed as a function of $\alpha$ and $\beta$ ). In the Riemann coordinates, the boundary conditions (4) rewrite as follows

$$
\begin{aligned}
& \beta^{+}=\frac{1}{1+\bar{Z}}\left(\alpha^{+}(1-\bar{Z})+2 \bar{Z} \beta^{-}\right), \\
& \alpha^{-}=\frac{1}{1+\bar{Z}}\left(2 \alpha^{+}+(\bar{Z}-1) \beta^{-}\right),
\end{aligned}
$$

where we have denoted the relative magnitude of the impedance as

$$
\bar{Z}=\left[\frac{c_{t}}{J G}\right]^{+} /\left[\frac{c_{t}}{J G}\right]^{-}
$$

The boundary condition (7) rewrites

$$
\frac{\partial \omega_{0}}{\partial t}=\frac{1}{I_{T D}}\left(\tau_{m}+\frac{G J}{c_{t}}\left(\beta(t, 0)-\omega_{0}(t)\right)\right) .
$$

In the case of the same material being used at both sides of the discontinuity, the only change is in the polar moment of inertia. That is, for a pipe-collar sections of e.g. steel, we have, following Fig. 3

$$
\bar{Z}=\frac{J_{c}}{J_{p}} .
$$

Note the meaning of (12)-(13) as reflections of incoming waves from both sides, as they are split into an upward and a downward travelling wave.

\subsection{Model validity}

The effectiveness of this modeling approach is explored in some detail in [1]. Here we illustrate this by briefly considering the open-loop fit of the model to full scale field data shown in Fig 4 and 5. Details of the numerical implementation is given in Appendix A.

In both the cases of Fig 4 and 5, the model accurately replicates the stick slip oscillations of the field data. The cases covers two qualitatively different behaviour of the stick slip, demonstrating a certain degree of versatility of the model. In particular we note that, for Fig. 4 where down-hole data is available, a good replication of the angular BHA velocity is also achieved. Down-hole data for the well in Fig. 5 is not available.

These fits were achieved by tuning the friction factors of the model to get the simulation results to match the data. The results in [1] verify that the model is capable to replicate the full scale field dynamics in most cases when this tuning is good. The goal of the present paper is to obtain an automated algorithm, the adaptive observer (a.k.a. soft sensor), which performs this tuning online. The design and testing of this adaptive observer is done in the following.

\section{Semi-lumped approximation}

In this section we derive a model for observer design by using a lumped approximation of the drill collar section following the approach of [3]. This amenable model approximation will be used for the observer design.

\subsection{Lumped BHA}

The approximation entails lumping the effect of the source term (11) into the lumped dynamics of the BHA. This is a reasonable approximation for many drill-strings as much of the torque acting on the drill string will come from stabilizers located in, or close to, the BHA [1]. The inertia of the lumped BHA is

$$
I_{B H A}=\rho L_{c} J_{c}
$$

and hence the angular velocity of the BHA is governed by

$$
\frac{\partial \omega_{L_{p}}}{\partial t}=\frac{1}{I_{B H A}}\left(\tau\left(t, x=L_{p}\right)-d(t)\right),
$$




\section{a)}

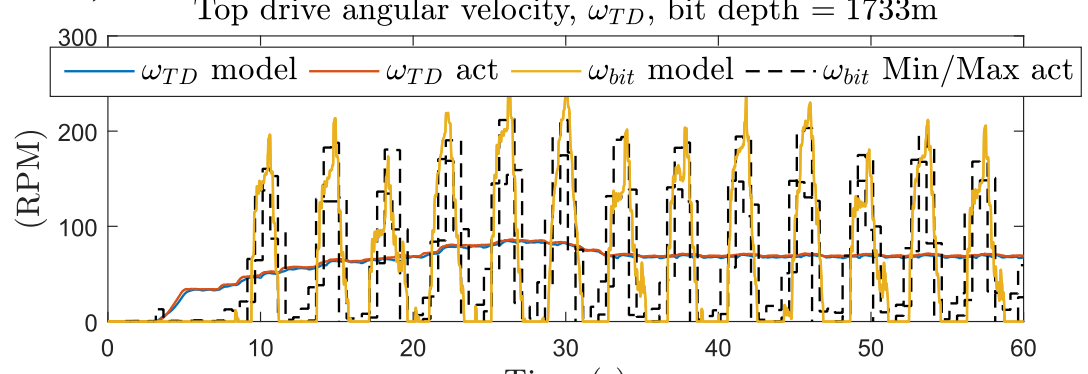

b)

Time $(\mathrm{s})$
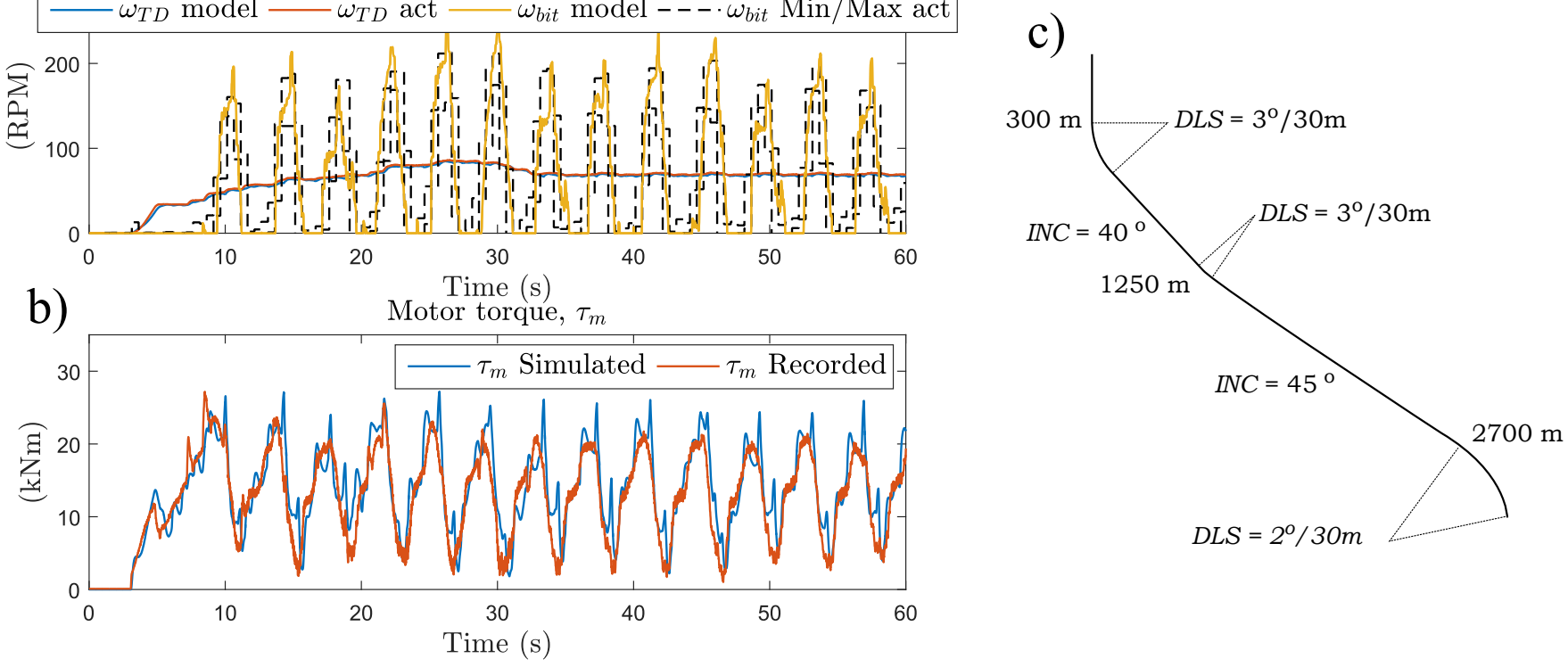

Figure 4: Recorded and simulated a) RPM and $\mathbf{b}$ torque of a drill-string at a bit depth of 1,733 $\mathrm{m}$ in a well with the survey shown in c), using the friction parameters: $\mu_{s}=0.34, \mu_{k}=0.187, \omega_{c}=19(\mathrm{RPM})$.

a)

Top drive angular velocity, $\omega_{T D}$, bit depth $=2506 \mathrm{~m}$

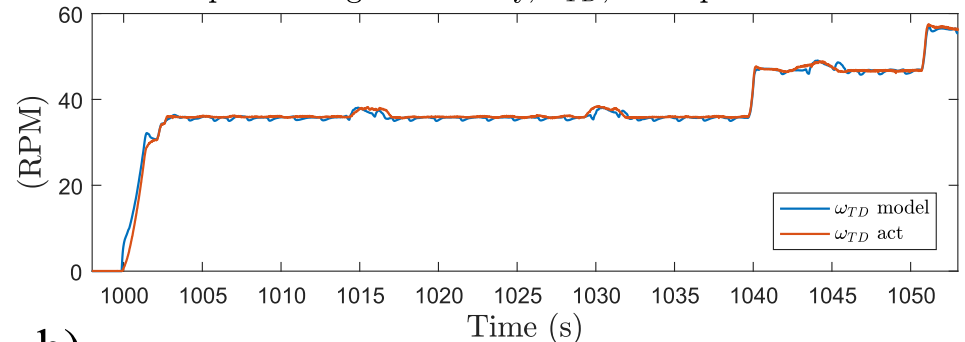

b)

Time $(\mathrm{s})$
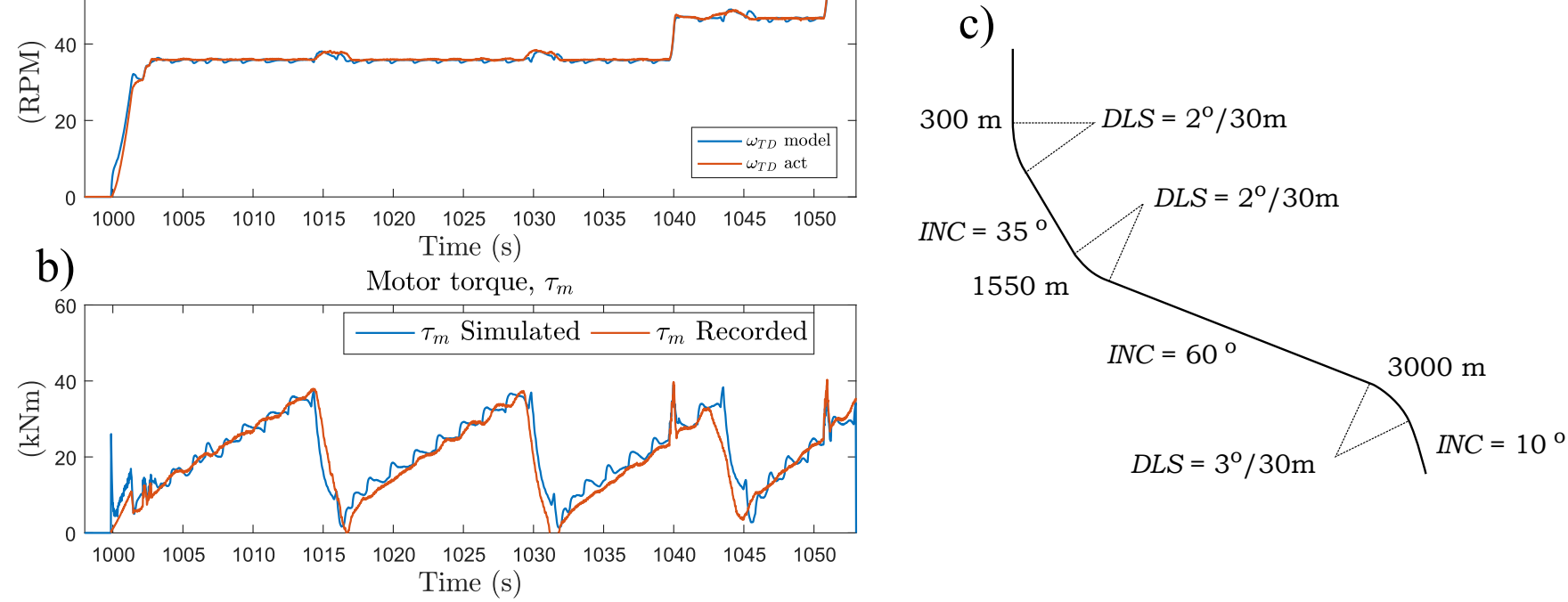

Figure 5: Recorded and simulated a) RPM and b) torque of a drill-string at a bit depth of 2,506 m in a well with the survey shown in c), using the friction parameters: $\mu_{S}=0.43, \mu_{k}=0.185, \omega_{c}=17$ (RPM). 
where $d(t)$ accounts for the now lumped effect of the distributed source term, i.e.:

$$
d(t) \approx \int_{0}^{L} S(\omega, x) .
$$

Here, (19) is meant for illustration and is not used directly. When we later employ the flat formulation, facilitated by this approximation, for estimation $d(t)$ will be treated as an uncertain disturbance.

Using this lumped approximation of BHA, we obtain what we will refer to as the semi-lumped formulation, given by the distributed wave-equation for the pipe section

$$
\begin{aligned}
& \frac{\partial \tau_{p}(t, x)}{\partial t}+J G \frac{\partial \omega_{p}(t, x)}{\partial x}=0, \\
& J_{p} \rho \frac{\partial \omega_{p}(t, x)}{\partial t}+\frac{\partial \tau_{p}(t, x)}{\partial x}=0,
\end{aligned}
$$

defined on $\left\{(t, x) \mid 0<t<T, \quad x \in\left[0, L_{p}\right]\right\}$ with the boundary conditions $\omega_{p}(t, x=0)=\omega_{0}, \omega_{p}\left(t, x=L_{p}\right)=\omega_{L_{p}}$ governed by

$$
\begin{aligned}
\frac{\partial \omega_{0}}{\partial t} & =\frac{1}{I_{T D}}\left(\tau_{m}-\tau_{p}(t, x=0)\right), \\
\frac{\partial \omega_{L_{p}}}{\partial t} & =\frac{1}{I_{B H A}}\left(\tau_{p}\left(t, x=L_{p}\right)-d(t)\right) .
\end{aligned}
$$

The fit of this approximation and how to quantify any resulting error is discussed in [3].

\subsection{In Riemann invariants}

Using the Riemann Variables $(\alpha, \beta)$ as states, the semilumped system (20)-(23) defined on $\{(t, x) \mid 0<t<$ $\left.T, \quad x \in\left[0, L_{p}\right]\right\}$, rewrites:

$$
\begin{aligned}
& \frac{\partial \alpha_{p}}{\partial t}(t, x)+c_{t} \frac{\partial \alpha_{p}}{\partial x}(t, x)=0, \\
& \frac{\partial \beta_{p}}{\partial t}(t, x)-c_{t} \frac{\partial \beta_{p}}{\partial x}(t, x)=0 .
\end{aligned}
$$

with the boundary conditions

$$
\begin{aligned}
\beta_{p}\left(t, L_{p}\right) & =2 \omega_{L_{p}}(t)-\alpha_{p}\left(t, L_{p}\right), \\
\alpha_{p}(t, 0) & =2 \omega_{0}(t)-\beta_{p}(t, 0), \\
\frac{\partial \omega_{0}}{\partial t}(t) & =a_{0}\left(-\omega_{0}(t)+\beta_{p}(t, 0)\right)+\frac{1}{I_{T D}} \tau_{m}(t) \\
\frac{\partial \omega_{L_{p}}}{\partial t}(t) & =a_{L_{p}}\left(-\omega_{L_{p}}(t)+\alpha_{p}\left(t, L_{p}\right)\right)-\frac{1}{I_{B H A}} d,
\end{aligned}
$$

where the frequency constants given as $a_{0}=\frac{G_{p} J_{p}}{c_{t} I_{T D}}$ and $a_{L_{p}}=$ $\frac{G_{p} J_{p}}{c_{t} I_{B H A}}$. They are expressed in seconds ${ }^{-1}$ and they repectively represent the inertia of the top-drive and BHA, relative to the line impedance of the pipe section of the drill string $\zeta_{p}=\frac{G_{p} J_{p}}{c_{t}}$. The solution to (24)-(25) can be written as the delay equations:

$$
\begin{aligned}
\alpha_{p}\left(t, x=L_{p}\right) & =\alpha_{p}\left(t-t_{D}, x=0\right) \\
\beta_{p}(t, x=0) & =\beta_{p}\left(t-t_{D}, x=L_{p}\right),
\end{aligned}
$$

where $t_{D}=L_{p} / c_{t}$. Thus, we note that this system is characterized by three time constants
$1 / a_{0}:$ Top drive time constant.

$1 / a_{L_{p}}$ : BHA time constant.

$t_{D}$ : Drill string travel time.

\section{Soft sensor (Observer) design}

The core of the approach is a 'soft-sensor' combining measurements from physical sensors with a model of the system dynamics to provide estimates of states and sideforces. The soft-sensor is based on an observer algorithm. Observers are dynamical systems whose states are the estimates of the aforementioned variables. The observer state dynamics are a combination of

- 'open-loop' terms based on the equations of physics describing the behaviour of the system.

- 'closed-loop' terms that correct the dynamics based on the value of the measurements. Typically, the value from the sensors are compared with their estimates from the observer, and a function of the discrepancy between the two are added as a correction term.

The task of design an observer is to engineer the combination of 'open-loop' and closed-loop correction terms. Often, the correction terms are linear function of the estimation error of the measured states. These correction terms are then used to update the estimated states as new measurement data become available. In our case, they appear in the observer dynamics as spatially-varying and lumped gains called observer gains. We will use the specific observer gains derived in [12] using a backstepping method which guarantees fast and robust convergence of the estimates assuming the model is correct ${ }^{1}$.

The soft sensor is adaptive in that it estimates the side forces and then updates the observer model kinetic or static friction factor itteratively for each time step depending on if the drill string BHA is stuck or sliding. The algorithm presented here is not able to simultaneously update the transition velocity $\omega_{c}$ in $(5)$, hence this value has to be fixed. We remark that it was shown in [1] that this transition velocity can have a significant impact on model bahvior in some cases, which might limit the performance of the proposed algorithm.

\subsection{Observer system equations}

The observer equations are given as a copy of the plant equations plus the correction terms. The measured output of the system corresponds to the top drive angular velocity $\omega_{0}$.

\footnotetext{
${ }^{1}$ More precisely, a proof of asymptotic convergence of the estimates to the true states is provided in [12] for the linearised dynamics, i.e. close to the constant homogeneous velocity profile.
} 
We denote an estimated variable with the $\hat{\imath}$ superscript. We define the measured estimation error as: $e=\hat{\omega}_{0}-\omega_{0}$. In the following $p_{\alpha}(\cdot), p_{\beta}(\cdot), p_{0}, p_{1}, P_{0}, P_{1}, l_{s}$ and $l_{k}$ denote the observer gains, which still have to be defined. The estimation of the top drive angular velocity is given by

$$
\dot{\hat{\omega}}_{0}=a_{0}\left(\hat{\beta}_{p}(t, 0)-\hat{\omega}_{0}\right)+\frac{1}{I_{T D}} \tau_{m}-p_{0} e,
$$

For the pipe section, the estimation of the Riemann invariant is given by

$$
\begin{aligned}
\frac{\partial \hat{\alpha}_{p}}{\partial t}(t, x)+c_{t} \frac{\partial \hat{\alpha}_{p}}{\partial x}(t, x) & =\hat{\mathcal{S}}_{p}(t, x)-p_{\alpha}(x) e, \\
\frac{\partial \hat{\beta}_{p}}{\partial t}(t, x)-c_{t} \frac{\partial \hat{\beta}_{p}}{\partial x}(t, x) & =\hat{\mathcal{S}}_{p}(t, x)-p_{\beta}(x) e .
\end{aligned}
$$

For the collar section, the estimation of the Riemann invariant is given by

$$
\begin{aligned}
\frac{\partial \hat{\alpha}_{c}}{\partial t}(t, x)+c_{t} \frac{\partial \hat{\alpha}_{c}}{\partial x}(t, x) & =\hat{\mathcal{S}}_{c}(t, x)-p_{1} e, \\
\frac{\partial \hat{\beta}_{c}}{\partial t}(t, x)-c_{t} \frac{\partial \hat{\beta}_{c}}{\partial x}(t, x) & =\hat{\mathcal{S}}_{c}(t, x)-p_{1} e .
\end{aligned}
$$

Finally, the boundary conditions are

$$
\begin{aligned}
\hat{\alpha}_{p}(t, 0) & =2 \hat{\omega}_{0}(t)-\hat{\beta}_{p}(t, 0)-P_{0} e \\
\hat{\beta}_{p}\left(t, L_{p}\right) & =\frac{\hat{\alpha}_{p}\left(t, L_{p}\right)(1-\bar{Z})+2 \bar{Z} \hat{\beta}_{c}\left(t, L_{p}\right)}{1+\bar{Z}}-P_{1} e \\
\hat{\alpha}_{c}\left(t, L_{p}\right) & =\frac{2 \hat{\alpha}_{p}\left(t, L_{p}\right)-\hat{\beta}_{c}\left(t, L_{p}\right)(1-\bar{Z})}{1+\bar{Z}} \\
\hat{\beta}_{c}(t, L) & =-\hat{\alpha}_{c}(t, L)
\end{aligned}
$$

The source term in each section are computed from the estimated states and friction factors

$$
\hat{\mathcal{S}}_{i}(t, x)=\frac{\hat{S}(t, x)}{J_{i} \rho}=k_{t}\left(\hat{\alpha}_{i}(t, x)+\hat{\beta}_{i}(t, x)\right)+\frac{1}{J_{i} \rho} \mathcal{F}(t, x),
$$

where $\mathcal{F}(t, x)$ is the inclusion given in (5), and the estimates of the friction factor is updated according to

$$
\begin{aligned}
& \dot{\hat{\mu}}_{s}(t)= \begin{cases}-l_{s} e, & \left|\hat{\omega}_{L_{p}}\right| \leq \omega_{c}, \\
0, & \left|\hat{\omega}_{L_{p}}\right|>\omega_{c},\end{cases} \\
& \dot{\hat{\mu}}_{k}(t)= \begin{cases}0, & \left|\hat{\omega}_{L_{p}}\right| \leq \omega_{c}, \\
l_{k} e, & \left|\hat{\omega}_{L_{p}}\right|>\omega_{c},\end{cases}
\end{aligned}
$$

Finally, the following saturation is used to improve robustness of the method:

$$
\hat{\mu}_{s}=\max \left(\hat{\mu}_{s}, \hat{\mu}_{k}\right) .
$$

The initial condition of (32)-(40) can be arbitrarily chosen.

\subsection{Semi-lumped approximation and error system}

We want to design the observer gains using the approach proposed in [12] to ensure the convergence of the observer state (solution of (32)-(40)) to the real state (solution of (9)-(15)). To do so, we need to rewrite the observer system in a suitable form (i.e. without the inclusion). This is done using the lumped approximation of the drill collar section introduced in Section 3.1. More precisely, the observer dynamics (32)-(40) can be rewritten

$$
\begin{aligned}
& \dot{\hat{\omega}}_{0}(t)=a_{0}\left(\hat{\beta}_{p}(t, 0)-\hat{\omega}_{0}(t)\right)+\frac{1}{I_{T D}} \tau_{m}-p_{0} e, \\
& \frac{\partial \hat{\alpha}_{p}}{\partial t}(t, x)+c_{t} \frac{\partial \hat{\alpha}_{p}}{\partial x}(t, x)=-p_{\alpha}(x) e \\
& \frac{\partial \hat{\beta}_{p}}{\partial t}(t, x)-c_{t} \frac{\partial \hat{\beta}_{p}}{\partial x}(t, x)=-p_{\beta}(x) e \\
& \dot{\hat{\omega}}_{L_{p}}(t)=a_{L_{p}}\left(\hat{\alpha}_{p}\left(t, L_{p}\right)-\hat{\omega}_{L_{p}}(t)\right)-\frac{1}{I_{B H A}} \hat{d}-p_{1} e,
\end{aligned}
$$

with the boundary conditions

$$
\begin{aligned}
\hat{\alpha}_{p}(t, 0) & =2 \hat{\omega}_{0}(t)-\hat{\beta}_{p}(t, 0)-P_{0} e, \\
\hat{\beta}_{p}\left(t, L_{p}\right) & =2 \hat{\omega}_{L_{p}}(t)-\hat{\alpha}_{p}\left(t, L_{p}\right)-P_{1} e,
\end{aligned}
$$

Similarly to what has been done in Section 3.1, the term $\hat{d}$ accounts for the now lumped effect of the distributed source term (obviously depending on the expression given in (41)). For the design of observer gains, we consider the term $d$ as a constant lumped disturbance, normalized by $\frac{I_{B H A}}{\int_{0}^{L} F_{N}(x) \mathrm{d} x}$ (thus, $\hat{d}$ is also assumed to be constant). Subtracting system (24)-(29) from system (45)-(50) and denoting the error variables with the $\tilde{\sim}$ superscript (i.e $\tilde{\alpha}_{p}=\hat{\alpha}_{p}-\alpha_{p}$ for instance), we get the following error system

$$
\begin{aligned}
& \dot{\tilde{\omega}}_{0}(t)=a_{0}\left(\tilde{\beta}_{p}(t, 0)-e\right)-p_{0} e \\
& \frac{\partial \tilde{\alpha}_{p}}{\partial t}(t, x)+c_{t} \frac{\partial \tilde{\alpha}_{p}}{\partial x}(t, x)=-p_{\alpha}(x) e, \\
& \frac{\partial \tilde{\beta}_{p}}{\partial t}(t, x)-c_{t} \frac{\partial \tilde{\beta}_{p}}{\partial x}(t, x)=-p_{\beta}(x) e, \\
& \dot{\tilde{\omega}}_{L_{p}}(t)=a_{L_{p}}\left(\tilde{\alpha}_{p}\left(t, L_{p}\right)-\tilde{\omega}_{L_{p}}(t)\right)-p_{1} e-\frac{1}{I_{B H A}} \tilde{d}, \\
& \dot{\tilde{d}}=0,
\end{aligned}
$$

with the boundary conditions

$$
\begin{aligned}
\tilde{\alpha}_{p}(t, 0) & =2 \tilde{\omega}_{0}(t)-\tilde{\beta}_{p}(t, 0)-P_{0} e, \\
\tilde{\beta}_{p}\left(t, L_{p}\right) & =2 \tilde{\omega}_{L_{p}}(t)-\tilde{\alpha}_{p}\left(t, L_{p}\right)-P_{1} e,
\end{aligned}
$$

\subsection{Control dual problem}

The backstepping approach proposed in [12] provides an explicit method to design a robust output feedback boundary control law for an ODE-PDE-ODE interconnection. However, it has been proved in [7] that the gains of such a control law correspond to the gain of the observer of the dual problem (this has actually been proved 
in the case of a system of $n+m$ PDEs, but the proof can be easily adjusted to an ODE-PDE-ODE interconnection). More precisely, adjusting the methods proposed in [7] we can prove that the system (51)-(57) has the same stability properties as those of the system defined on $\{(t, x) \mid \quad 0<t<T, \quad x \in[0,1]\}$ by

$$
\begin{aligned}
& \frac{\partial \psi}{\partial t}(t, x)-\frac{c_{t}}{L_{p}} \frac{\partial \psi}{\partial x}(t, x)=0 \\
& \frac{\partial \phi}{\partial t}(t, x)+\frac{c_{t}}{L_{p}} \frac{\partial \phi}{\partial x}(t, x)=0 \\
& \dot{\chi}_{0}(t)=2 \frac{c_{t}}{L_{p}} \psi(t, 0)-a_{0} \chi_{0}-p_{0} \chi_{0}(t)-p_{1} \chi_{1}(t)-P_{0} c_{t} \psi(t, 0) \\
& -P_{1} c_{t} \phi\left(t, L_{p}\right)-\int_{0}^{L_{p}} p_{\alpha}(\xi) \psi(t, \xi)+p_{\beta}(\xi) \phi(t, \xi) d \xi \\
& \dot{\chi}_{1}(t)=2 \frac{c_{t}}{L_{p}} \phi(t, 1)-a_{L_{p}} \chi_{1}(t) \\
& \dot{\chi}_{2}(t)=-\frac{1}{I_{B H A}} \chi_{1}(t)
\end{aligned}
$$

with the boundary conditions

$$
\begin{gathered}
\psi(t, 1)=-\phi(t, 1)+\frac{L_{p}}{c_{t}} a_{L_{p}} \chi_{1}(t), \\
\phi(t, 0)=-\phi(t, 0)+\frac{L_{p}}{c_{t}} a_{0} \chi_{0}(t)
\end{gathered}
$$

This new system (which is the adjoint of (51)-(57)) has exactly the structure which is considered in [12]. This immediately gives us the expression of the observer gains (we choose to not rewrite them here for sake of clarity). More precisely, these observer gains only depends on three tuning parameters (namely, $\kappa_{1}, z$ and $p$ ) that can be tuned to shape the observer top-side reflection and place the poles of the down-hole "error system". Considering the adaptive update law, the gain $l_{k}$, is derived from the observer assuming a constant lumped disturbance, and then normalized by $\frac{I_{B H A}}{\int_{0}^{L} F_{N}(x) \mathrm{d} x}$. The gain for the update law of the static friction factor is then chosen to be two times this $l_{s}=2 l_{k}$. With these gains we can ensure the convergence of the observer states to the real states in presence of a constant disturbance. One must be aware that this assumption is only an approximation and does not hold in the real case. However, due to the robustness properties of the observer (see [12] for details) we can still have the convergence of the different states to their real values when considering the real inclusion term. Considering the estimations of the friction terms, we do not have any guarantee of convergence, but, due to the robustness properties of the observer, they should converge close to the real friction terms. This is experimentally validated in the next section.

\section{Field Application}

The observer detailed in the previous section may be used to provide online parameter estimation for the friction

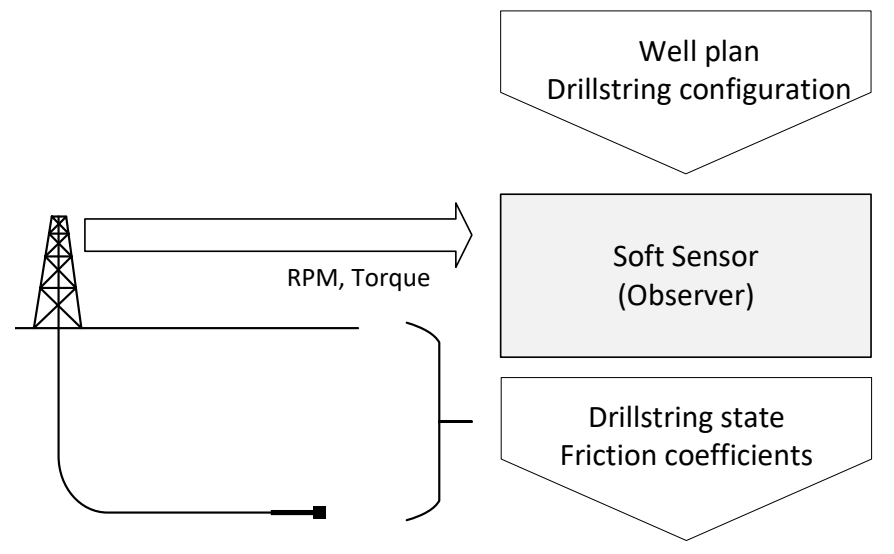

Figure 6: Flow of data from the surface sensors, into the estimation algorithm and the resulting estimated output.

parameters of the drillstring model presented in Section 2 but can also be used as a method to estimate BHA rotation and torque. This can be of particular usefulness in directional drilling scenarios where real-time estimation of tool face angle - BHA angular orientation - is essential, and in feedforward stick-slip mitigation systems.

The envisioned industrial implementation of the estimatino algorithm is briefly described in this section. The flow of data is show in Figure 6 and a flowchart describing the process is presented in Figure 7.

Output of the estimation algorithm may include an estimate of the drillstring state as a function of measure depth, and a time series of the friction coefficient, estimated drillstring twist and the soft sensor (observer) gains.

We now consider a test of the estimation algorithm (32)(43). We want to evaluate the real time estimation of the friction coefficients and of the BHA and top drive rotation in two different situations: (1) comparison against a simulation model (2) comparison against field data. In each situation, we run the observer using high frequency measurements $(100 \mathrm{~Hz})$ but also downsampled $5 \mathrm{~Hz}$ and 1 $\mathrm{Hz}$ measurements. These examples are of particular note since a majority of supervisory control systems currently deployed on drilling rigs in the field operate at 5 to $10 \mathrm{~Hz}$.

\subsection{Test against simulation model}

We test our observer against the simulation model described in [5] with the wellbore survey shown in Figure 8, using the numerical implementation described in Appendix A. The kinetic friction is chosen to be equal to 0.187, while the static friction is chosen to be equal to 0.6 which is similar to values reported using traditional friction tests in the field. The well represents a simple build 


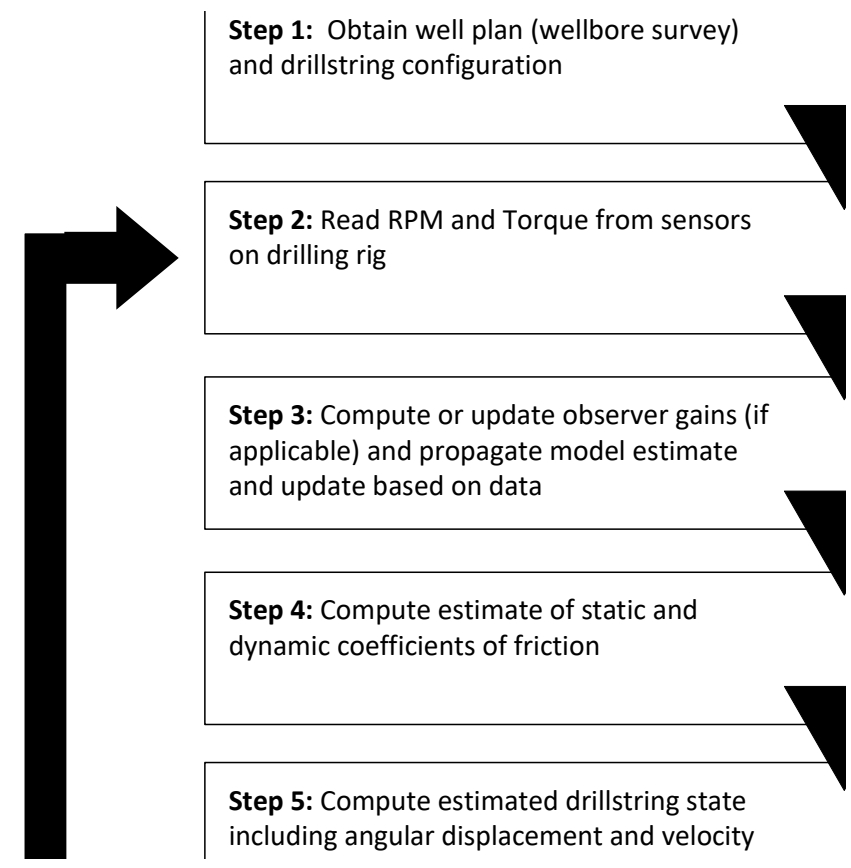

Figure 7: Flow chart describing the flow of information and steps used in the potential application.

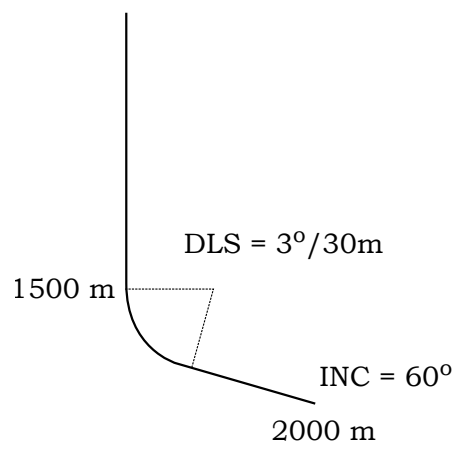

Figure 8: Wellbore survey for the simulation model.

and hold well used throughout the world. More discussion of this synthetic example may be found in [1].

We have pictured in Fig. 9 the real value of the BHA angular velocity and of the top drive angular velocity as well as the estimations obtained using the observer in three different situations. In the first plot, the measurements are available every $0.01 \mathrm{~s}(100 \mathrm{~Hz})$, while in the two next plots they are only available every $0.2 \mathrm{~s}(5 \mathrm{~Hz})$, resp. $1 \mathrm{~s}(1 \mathrm{~Hz}))$. This means that for the cases with lower sampling rates, the observer correction term is less frequently updated, which we would expect to potentially reduce convergence rate or accuracy. As is seen in Fig. 9, the 100 and $5 \mathrm{~Hz}$ cases show a rapid convergence of the estimated states to the correct trajectories, while at $1 \mathrm{~Hz}$ sampling rate the performance has started to degrade.

We have pictured in Fig. 10, the estimation given by the observer of the friction parameters for different starting points in the three different situations $(100 \mathrm{~Hz}, 5 \mathrm{~Hz}$ and $1 \mathrm{~Hz}$ measurements). In the two first cases, we have a rapid convergence of the estimations regardless of the initial guess. However, the convergence value (in particular for the static friction term) may not exactly correspond to the exact one (even if it remains close). In the last case ( $1 \mathrm{~Hz}$ measurements), the estimations of the friction terms oscillate around the exact value. The estimations remain good however.

\subsection{Test on field data}

We now test our observer against field data obtained from an onshore well with the wellpath shown in Fig. 4. Surface rpm and torque data was recorded at $100 \mathrm{~Hz}$ using a high frequency data recorder and downhole data in the BHA was recorded on a memory tool as a 10 second windowed minimum, maximum and average value. RPM data was obtained using an encoder at surface and via magnetometer/accelerometer data downhole. Surface torque data was obtained from current integration in the inverter controller the topdrive and pipe torque was inferred by removing inertial acceration torque. Top drive inertia was obtained using a chirp test. We have pictured in Fig. 11 the real mean value of the BHA angular velocity and of the top drive angular velocity as well as the estimations obtained using the observer, again for the three cases of $100 \mathrm{~Hz}, 5 \mathrm{~Hz}$ and $1 \mathrm{~Hz}$ data sampling rates. Once again, the observer is run at a frequency of $100 \mathrm{~Hz}$, possibly using the same value of the output for successive iterations in the two latter cases. In the three cases, the estimations provided by the observer tends to convergence to the real values. Note that the field data can be unsynchronized and that we don't take here the possible offset into account. Then we cannot really compare the phases of the field data/estimated angular velocities but only their respective magnitudes.

We have pictured in Fig. 12 the estimation given by the observer of the friction parameters for different starting points in the three different situations $(100 \mathrm{~Hz}, 5 \mathrm{~Hz}$ and $1 \mathrm{~Hz}$ measurements). In the three cases the estimations converge to the same values ( 0.6 for the static friction and 0.35 for the kinetic friction) regardless of the initial guess.

\section{Conclusion}

We have proposed a model and algorithm to estimate both drill bit velocity and friction factors while drilling, using only surface measurements. The approach relies on an observer designed for the linearized system, combined with an update law of the static and kinemtic friction factors used in a nonlinear coulomb-friction model. The estimation is robust to poor initial estimates of the states and parameter, as have been illustrated on both synthetic and field data. The adaptive estimation of the friction factors enables the states estimates of the observer to converge (close to) actual values in real time. This means that we are able to continously monitor the downhole behavior of 
a)

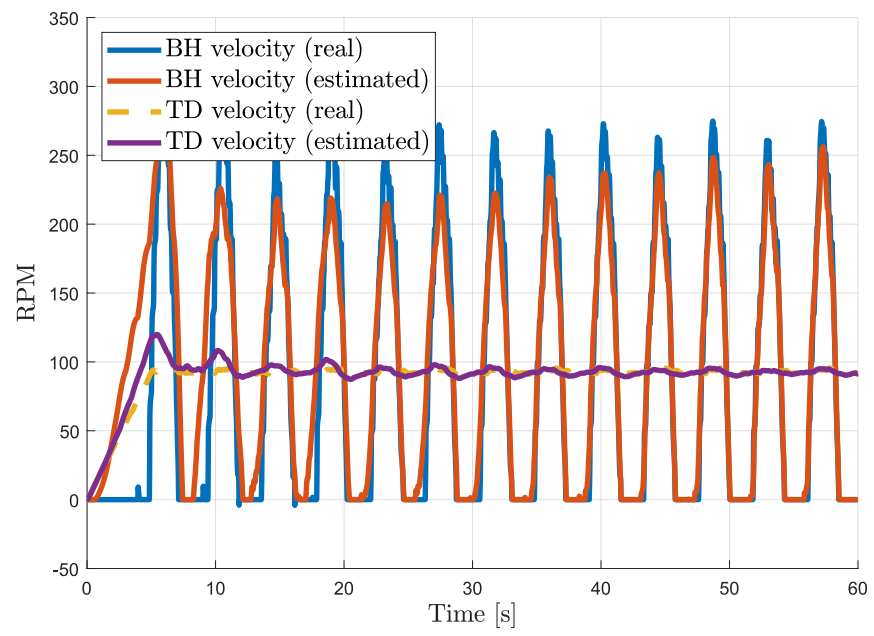

b)

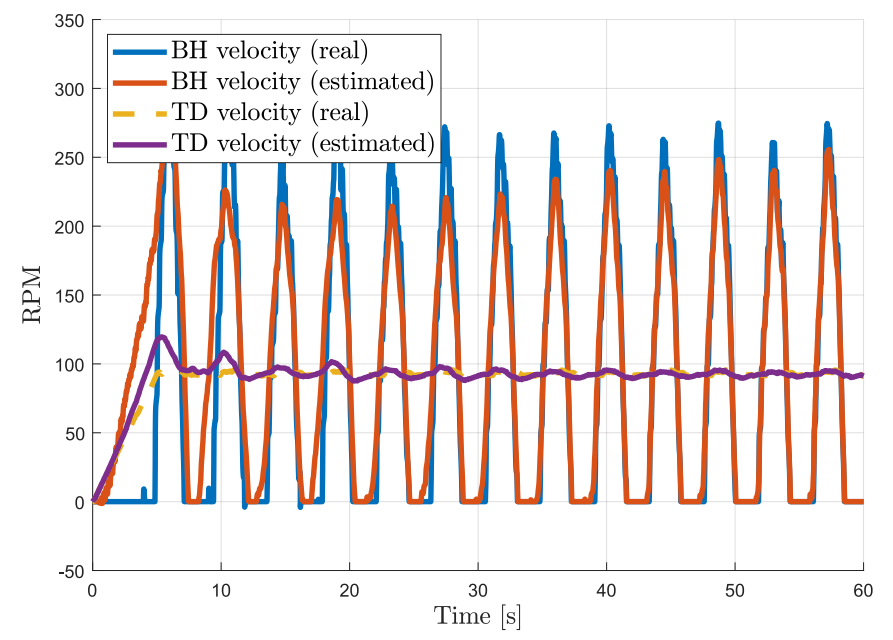

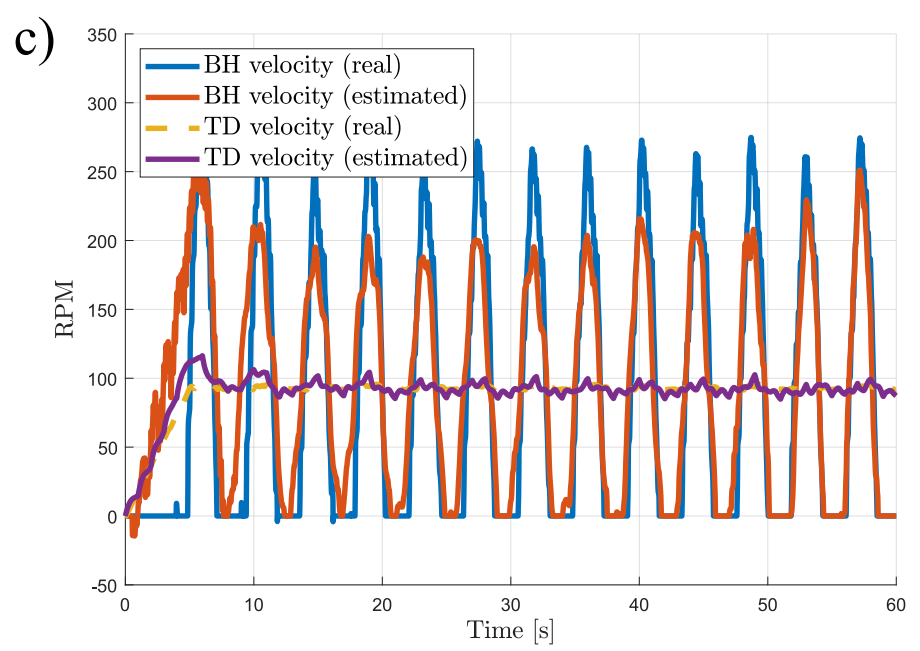

Figure 9: Time evolution of the real and estimated angular velocity in the test against simulation model for, a) $100 \mathrm{~Hz}, \mathbf{b}) 5 \mathrm{~Hz}$ and c) $1 \mathrm{~Hz}$ measurements.

the drill string, specifically the dynamic angular velocity and torques, even when drilling through stick slip. Furthermore, we envision that these state and parameter estimates could enable measures to counteract unwanted drill string behaviour, e.g. using a techniques such as proposed in [3], and represent a step towards automated closed loop geosteering.

\section{Acknowledgments}

This work was in part supported by the Research Council of Norway, ConocoPhillips, AkerBP, Statoil and Wintershall through the research center DrillWell (203525/O30) at IRIS, and by the Marie Skłodowska-Curie (MSCA) - Individual Fellowships through the Robust Estimation and Control of Infinite Dimensional Systems (RECIDS) Project. Additional support came from the University of Calgary's Canada First Research Excellence Fund Program, the Global Research Initiative in Sustainable Low Carbon Unconventional Resources.

\section{Appendix A. Numerical implementation}

In the numerical implementation of the model the wave equation (1),(2) is transformed into transport equations discretized using a first order upwind scheme. This choice is made to ensure numerical robustness and to avoid spurious oscillations, as higher order schemes perform poorly due to the temporal discontinuities introduced by the distributed differential inclusions which are used to represent the Coulomb friction. Numerical accuracy can then be ensured by having a sufficiently fine spatial grid, and this is an amenable approach due to the fact that simulation speed is not of critical importance for the present study.

In all simulations a spatial grid of 500 cells is used for the drill string and the time-step is chosen to enforce the Courant-Friedrichs-Lewy (CFL) condition [10].

In the numerical treatment of the model, $\mathcal{F}$ is implemented as follows. For cell size $\Delta x$ and and time step $\Delta t$, 
and at cell \# $j$ and time step \# $k$

$$
\begin{aligned}
\tilde{\mathcal{F}}_{j}^{k}= & \frac{1}{2 \Delta t}\left(\alpha_{j}-c_{t} \frac{\Delta t}{\Delta x}\left(\alpha_{j}-\alpha_{j-1}\right)+\right. \\
& \left.\beta_{j}+c_{t} \frac{\Delta t}{\Delta x}\left(\beta_{j+1}-\beta_{j}\right)+\Delta t k_{t}\left(\alpha_{j}^{k}+\beta_{j}^{k}\right)\right),
\end{aligned}
$$

and limited by

$$
\mathcal{F}_{j}^{k}= \begin{cases}\operatorname{sgn}\left(\tilde{\mathcal{F}}_{j}^{k}\right) \min \left(\left|\tilde{\mathcal{F}}_{j}^{k}\right|, \mu_{s} F_{N}\right), & \frac{\left|\alpha_{j}^{k}+\beta_{j}^{k}\right|}{2} \leq \omega_{c} \\ \operatorname{sgn}\left(\tilde{\mathcal{F}}_{j}^{k}\right) \min \left(\left|\tilde{\mathcal{F}}_{j}^{k}\right|, \mu_{k} F_{N}\right), & \frac{\left|\alpha_{j}^{k}+\beta_{j}^{k}\right|}{2}>\omega_{c}\end{cases}
$$

The model is updated with an upwind scheme according to

$$
\begin{aligned}
& \alpha_{j}^{k+1}=\alpha_{j}^{k}-c_{t} \frac{\Delta t}{\Delta x}\left(\alpha_{j}^{k}-\alpha_{j-1}^{k}\right)-\Delta t k_{t}\left(\alpha_{j}^{k}+\beta_{j}^{k}\right)-\mathcal{F}_{j}^{k} \\
& \beta_{j}^{k+1}=\beta_{j}^{k}-c_{t} \frac{\Delta t}{\Delta x}\left(\beta_{j}^{k}-\beta_{j+1}^{k}\right)-\Delta t k_{t}\left(\alpha_{j}^{k}+\beta_{j}^{k}\right)-\mathcal{F}_{j}^{k} .
\end{aligned}
$$

\section{References}

[1] U. J. Aarsnes and R. J. Shor. Torsional vibrations with bit off bottom: Modeling, characterization and field data validation. Journal of Petroleum Science and Engineering, 163:712-721, apr 2018.

[2] U. J. F. Aarsnes and O. M. Aamo. Linear stability analysis of self-excited vibrations in drilling using an infinite dimensional model. Journal of Sound and Vibration, 360:239-259, jan 2016.

[3] U. J. F Aarsnes, F. Di Meglio, and R. J. Shor. Avoiding stick slip vibrations in drilling through startup trajectory design. Journal of Process Control, 70:24-35, oct 2018.

[4] U J F Aarsnes and R J Shor. Stick-slip and torsional friction factors in inclined wellbores. In MATEC Web of Conferences, volume 148, 2018.

[5] U. J. F. Aarsnes and N. van de Wouw. Axial and torsional self-excited vibrations of a distributed drill-string. Submitted, 2018.

[6] U. J. F. Aarsnes and N. van de Wouw. Dynamics of a distributed drill string system: Characteristic parameters and stability maps. Journal of Sound and Vibration, 417:376-412, mar 2018.

[7] J. Auriol and F. Di Meglio. Two-sided boundary stabilization of heterodirectional linear coupled hyperbolic pdes. IEEE Transactions on Automatic Control, 63(8):2421-2436, 2018.

[8] J. F. Brett, A. D. Beckett, C. A. Holt, and D. L. Smith. Uses and Limitations of Drillstring Tension and Torque Models for Monitoring Hole Conditions. SPE Drilling Engineering, 4(03):223229 , sep 1989.

[9] E. Cheng and M. A. Polak. Theoretical model for calculating pulling loads for pipes in horizontal directional drilling. Tunnelling and Underground Space Technology, 22(5-6):633-643, 2007.

[10] R. Courant, K. Friedrichs, and H. Lewy. On the partial difference equation of mathematical physics. IBM journal of Research and Development, 11(215-234):32-74, 1967.

[11] E. Detournay and P. Defourny. A phenomenological model for the drilling action of drag bits. International Journal of Rock Mechanics and Mining Sciences \& Geomechanics Abstracts, 29(1):13-23, 1992.

[12] F. Di Meglio, P.-O. Lamare, and U. J. F. Aarsnes. Robust output feedback stabilization of an ODE-PDE-ODE interconnection. Submitted, (October):1-11, 2018.
[13] A F Filippov. Differential Equations with Discontinuous Righthand Sides. Springer Science \& Business Media, 1988.

[14] C. Germay, V. Denoël, and E. Detournay. Multiple mode analysis of the self-excited vibrations of rotary drilling systems. Journal of Sound and Vibration, 325(1-2):362-381, aug 2009.

[15] G. W. Halsey, A. Kyllingstad, T. V. Aarrestad, and D. Lysne. Drillstring Vibrations: Comparison Between Theory and Experiments on a Full-Scale Research Drilling Rig. In SPE/IADC Drilling Conference, number IADC/SPE 14760, pages 311-321. Society of Petroleum Engineers, apr 1986.

[16] R. J. LeVeque. Finite volume methods for hyperbolic problems. Cambridge university press, 2002.

[17] S. Menand. AADE-13-FTCE-21 Borehole Tortuosity Effect on Maximum Horizontal Drilling Length Based on Advanced Buckling Modeling. In AADE National Technical Conference and Exhibition, Oklahoma, 2013.

[18] T. Richard, C. Germay, and E. Detournay. Self-excited stickslip oscillations of drill bits. Comptes Rendus Mécanique, 332(8):619-626, aug 2004.

[19] L. P. Skyles, Y. Amiraslani, and J. Wilhoit. Converting static friction to kinetic friction to drill further and faster in directional holes. In IADC/SPE Drilling Conference and Exhibition, pages 1-16, San Diego, California, USA, 2012.

[20] P. Weijermans, J. Ruszka, H. Jamshidian, and M. Matheson. Drilling with Rotary Steerable System Reduces Wellbore Tortuosity. In SPE/IADC Drilling Conference. Society of Petroleum Engineers, apr 2001.

[21] D. Zhao, S. Hovda, and S. Sangesland. Abnormal Down Hole Pressure Variation by Axial Stick-Slip of Drillstring. Journal of Petroleum Science and Engineering, 145:194-204, 2016. 
a)

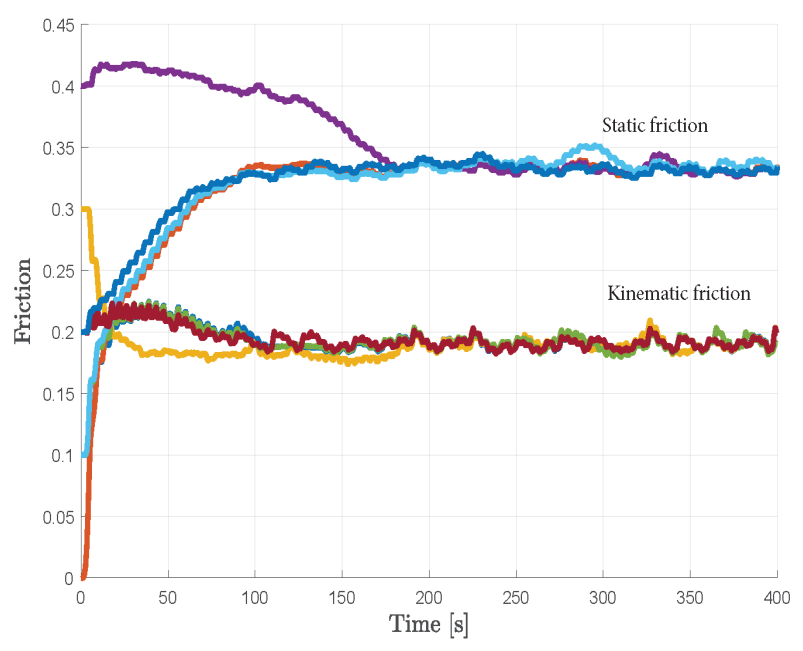

b)

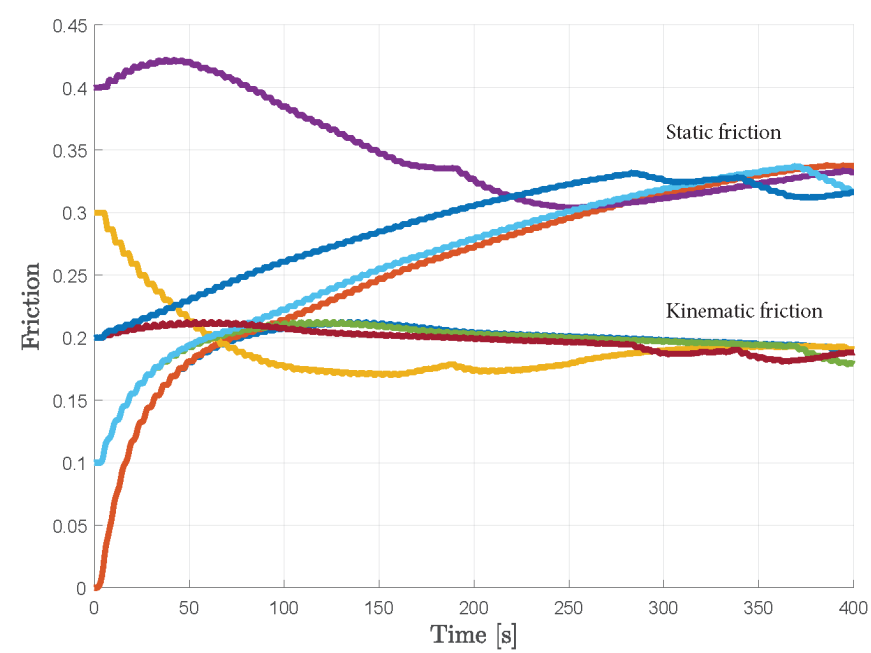

c)

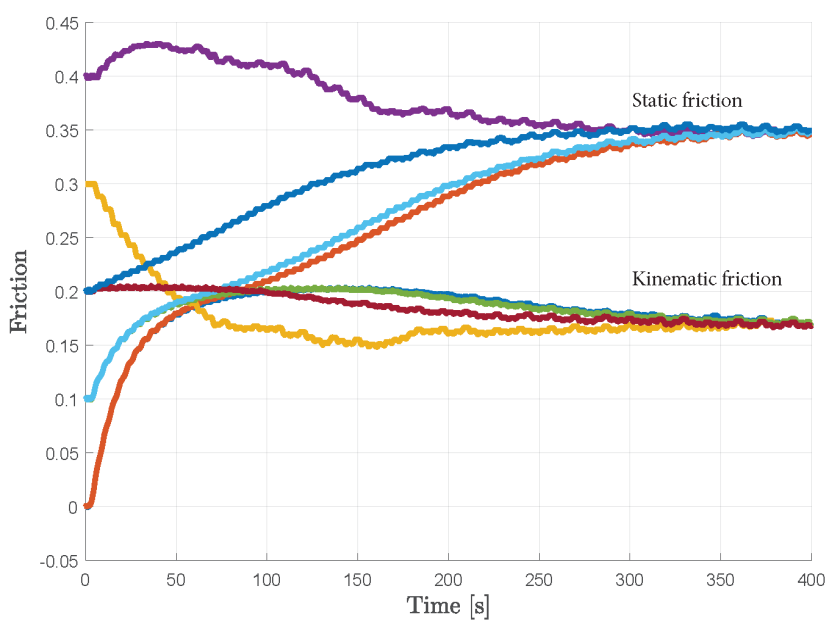

Figure 10: Estimation of static and kinetic friction factors for different initial conditions in the test against simulation model for, a) $100 \mathrm{~Hz}$, b) $5 \mathrm{~Hz}$ and c) $1 \mathrm{~Hz}$ measurements. 
a)

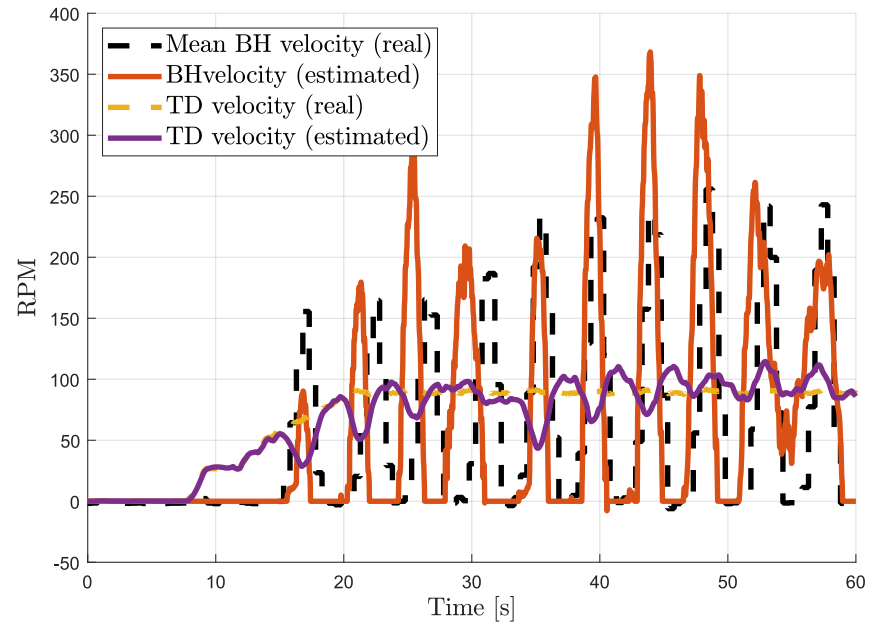

b)

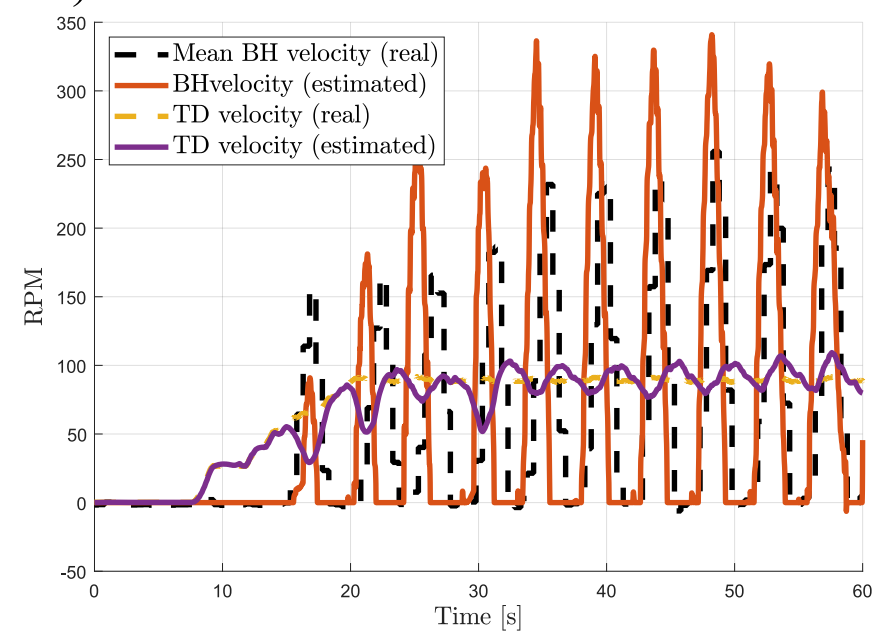

c)

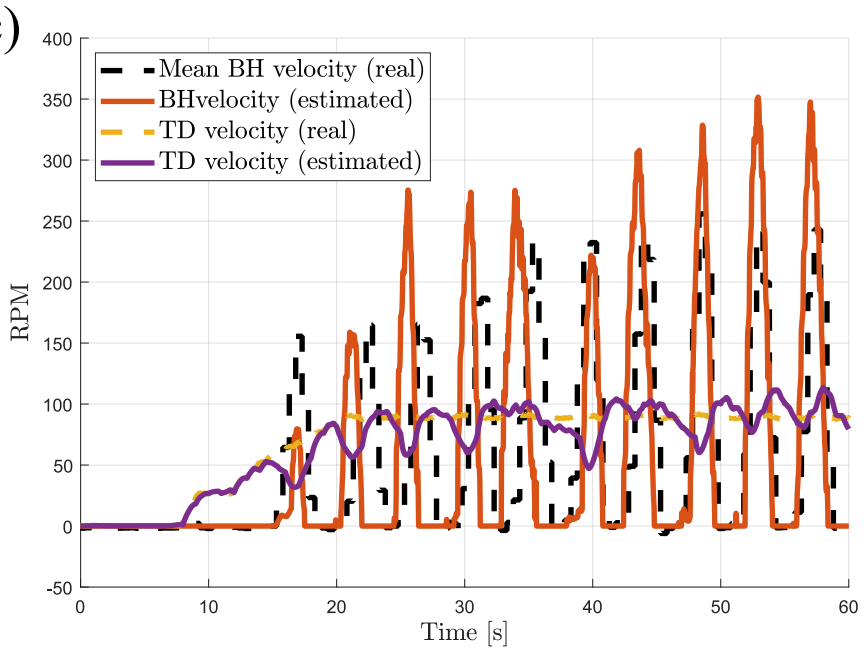

Figure 11: Real and estimated angular velocity of the BHA and the top drive in the test against field data for, respectively, a) $100 \mathrm{~Hz}, \mathbf{b )} 5 \mathrm{~Hz}$ and c) $1 \mathrm{~Hz}$ measurements. 
a)

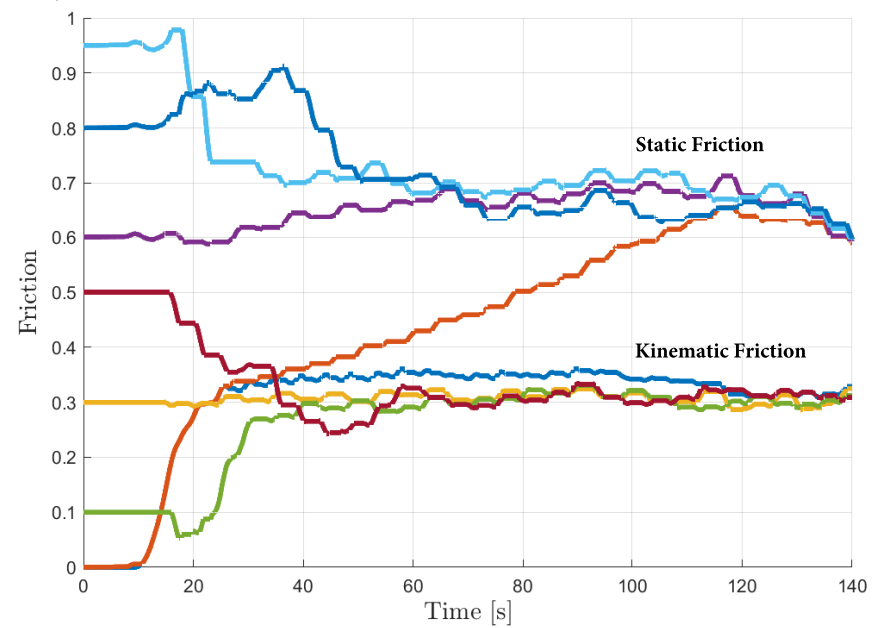

b)

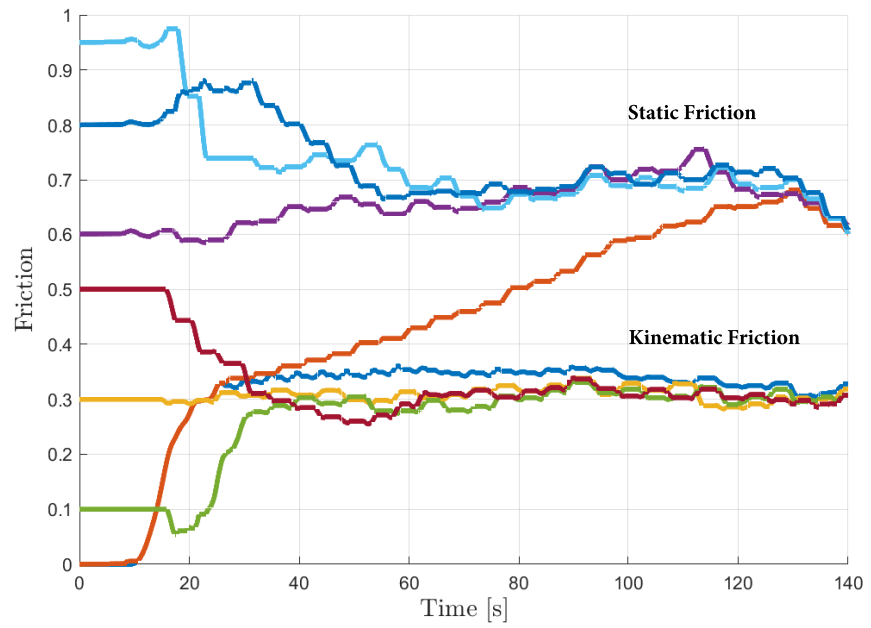

c)

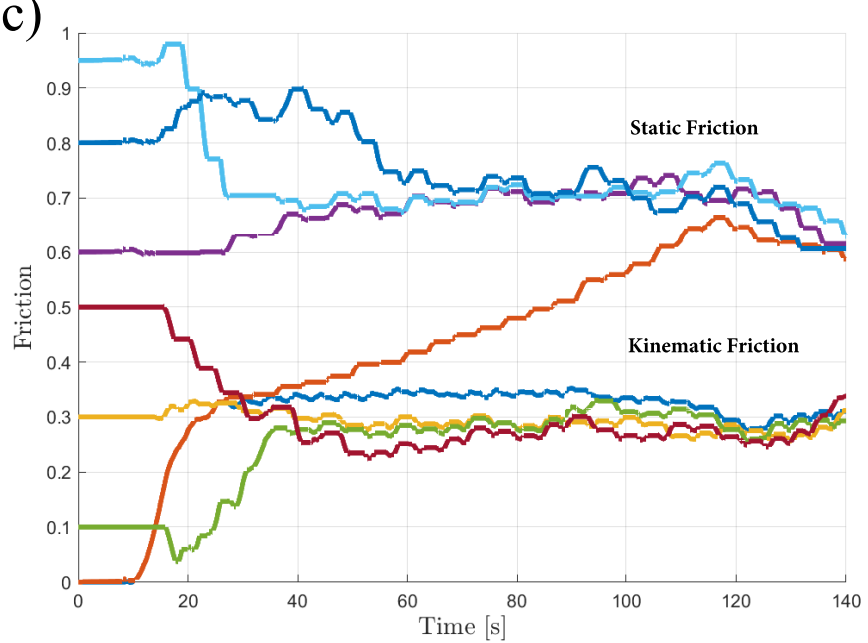

Figure 12: Estimation of static and kinetic friction factors for different initial conditions in the test against field data for, a) 100 $\mathrm{Hz}$, b) $5 \mathrm{~Hz}$ and c) $1 \mathrm{~Hz}$ measurements. 